\title{
Terapias para disminuir la sensibilidad por blanqueamiento dental
}

\author{
Therapies to decrease tooth whitening sensitivity \\ Kelly Achachao Almerco ${ }^{1, a}$, Lidia Yileng Tay Chu Jon 1,b
}

\section{RESUMEN}

La complicación más comun del tratamiento de blanqueamiento dental es la sensibilidad dental, ésta ocurre por la los radicales libres que se liberan a partir del peróxido de hidrógeno, principal agente de los productos blanqueadores, atraviesan el esmalte, la dentina y llegan a la pulpa dental produciendo el dolor. Existen diferentes terapias utilizadas antes, durante y después para disminuir esta sensibilidad dental, como el uso de agentes que actúan inhibiendo la repolarización del nervio como el nitrato de potasio y los que obliteran los túbulos entre ellos como el fosfato de calcio amorfo, silicato de calcio y sodio, gluconato de calcio, hidroxiapatita, GLUMA y flúor; existen otras terapias alternativas como el uso de antiinflamatorios, antioxidantes y aplicación de láser, las cuales también han sido revisadas en la literatura. La siguiente revisión tiene como objetivo mostrar la evidencia científica sobre los diferentes métodos para disminuir la sensibilidad por blanqueamiento dental.

PALABRAS CLAVE: blanqueamiento de dientes, desensibilizantes dentinarios, terapéutica. DECS

\section{SUMMARY}

The most common side effect in tooth bleaching is tooth sensitivity because of the pulp reaction to free radicals produced by the hydrogen peroxide used in bleaching products. Different therapies have been used before, during and after to reduce tooth sensitivity, agents that block nerve repolarization such as potassium nitrate and agents that block dentinal tubules: amorphous calcium phosphate, calcium sodium phosilicate, calcium gluconate, hydroxyapatite, GLUMA and fluor; alternative therapies such as the use of anti-inflammatory, antioxidants and laser application which have also been mentioned in the literature. The aim of this review is to show the scientific evidence on the different methods to decrease the sensitivity on tooth bleaching.

KEY WORDS: tooth bleaching, dentin desensitizing agents, therapeutics. MESH 


\section{INTRODUCCIÓN}

La búsqueda de tratamientos estéticos aumentó en los últimos años y con ello el blanqueamiento dental se ha convertido en un procedimiento popular (1), cuyo agente activo es el peróxido de hidrógeno que actúa penetrando la estructura del diente, quebrando las moléculas cromóforas por un mecanismo de óxido reducción (2-3).

A pesar de que el blanqueamiento dental es altamente efectivo, biológicamente seguro y mínimamente invasivo para despigmentar los dientes, se ha reportado como efecto adverso la sensibilidad dental en un 55 $\%$ de la población (4) y de estos el $14 \%$ no llega a completar el tratamiento (5).

Esta sensibilidad puede persistir hasta 4 días después de finalizado el procedimiento (6); los mecanismos responsables de la sensibilidad dental por blanqueamiento no han sido totalmente deducidos, sin embargo la sensación es posiblemente una indicación de la respuesta pulpar al peróxido de hidrógeno y sus radicales libres (7).

Por esta razón se han desarrollado agentes desensibilizantes para ayudar a reducir la duración e intensidad de la sensibilidad dental causada por el blanqueamiento dental, ya sea por despolarización de las fibras nerviosas, disminuir los cambios en la morfología del esmalte o promover su remineralización (8).

Actualmente no se ha encontrado una terapia universalmente aceptada que alivie completamente los síntomas de sensibilidad por blanqueamiento dental, aunque existe una variedad de productos que han sido formulados para su manejo los cuales han demostrado distintos grados de efectividad.

Considerando que la sensibilidad dental es el principal efecto adverso del blanqueamiento dental y existe una gran variedad de productos en diferentes presentaciones, la presente revisión tuvo como objetivo analizar la evidencia científica sobre los diferentes métodos para disminuir la sensibilidad por blanqueamiento dental.

\section{MATERIAL Y MÉTODOS}

La estrategia de búsqueda desarrollada para la revisión se llevó a cabo en las bases de datos: Pubmed, Lilacs, BBO, Scielo y Scopus. Las palabras claves utilizadas fueron (dentin desensitizing agents and tooth bleaching) se incluyeron estudios realizados en seres humanos de tipo ensayos clínicos, estudios observacionales, revisiones sistemáticas y un estudios in vitro, que evaluaron los métodos para disminuir la

Tabla 1. Terapias desensibilizantes revisadas

\begin{tabular}{|c|c|c|}
\hline TERAPIA DESENSIBILIZANTE & MECANISMO DE ACCIÓN & INDICACIÓN DE USO \\
\hline Nitrato de potasio & Altera la actividad neural de la pulpa & $\begin{array}{l}\text { Gel a base de nitrato de potasio } 5 \%: 10 \\
\text { min antes del blanqueamiento } \\
\text { Dentífricos: dos semanas antes, durante y } \\
\text { después del blanqueamiento. }\end{array}$ \\
\hline Flúor & Remineralización del esmalte y dentina & Antes y durante el blanqueamiento \\
\hline Gluma & Obliteración de los túbulos dentinarios & $\begin{array}{l}\text { Aplicación tópica después del } \\
\text { blanqueamiento }\end{array}$ \\
\hline $\begin{array}{l}\text { Fosfato de cálcio amorfo (ACP) } \\
\text { Fosilicato de calcio y sodio }\end{array}$ & Obliteración del os túbulos dentinarios & $\begin{array}{l}\text { Se encuentra dentro de la composición } \\
\text { del gel blanqueador }\end{array}$ \\
\hline Hidroxiapatita & Obliteración de los túbulos dentinarios & $\begin{array}{l}\text { Se aplica la pasta de } 3 \text { min a } 5 \text { min } \\
\text { después de la aplicación del gel } \\
\text { blanqueador }\end{array}$ \\
\hline $\begin{array}{l}\text { Antiinflamatorios } \\
\text { - Ibuprofeno }\end{array}$ & Inhibición de COX 1 y 2 & No se indica el uso \\
\hline - Eterocoxib & Inhibición selectiva de COX 2 & No se indica el uso \\
\hline Ácido Ascórbico & Antioxidante & No se indica el uso \\
\hline Laser & Despolariza las membranas nerviosas & No se indica el uso \\
\hline
\end{tabular}


sensibilidad por blanqueamiento dental.

Al combinar las búsquedas y filtrar solo los documentos en inglés y los que fueron realizados en humanos se generaron 100 artículos de los cuales 37 estuvieron relacionados con el tema de revisión.

Las terapias desensibilizantes encontradas en la literatura son las siguientes (tabla 1):

\section{a. El nitrato de potasio}

Puede ser usado antes, durante y después del blanqueamiento dental para minimizar la sensibilidad dental, ya que reduce la excitabilidad de las fibras nerviosas presentes en la pulpa evitando la repolarización de las terminaciones nerviosas previniendo que respondan a nuevos estímulos; este producto puede encontrarse dentro de la composición de dentífricos, geles desensibilizantes y en algunos productos blanqueadores (9).

Se ha demostrado que el nitrato de potasio ofrece la mayor eficacia en el tratamiento de desensibilización. ${ }^{8}$ Diversos estudios demuestran que el uso de gel de nitrato de potasio antes del blanqueamiento es efectivo, en el estudio de Tay et al., del 2009 (10), se evaluaron en pacientes sanos el uso de desensibilizantes a base de nitrato de potasio al $5 \%$ + fluoruro de sodio $2 \%$ durante 10 minutos antes del blanqueamiento con peróxido de hidrógeno al 35\% mostrando que el nitrato de potasio reduce el riesgo de presentar sensibilidad y si el paciente tuviese sensibilidad, esta sería leve; en otro estudio similar Reis et al., en el 2011 evaluaron los mismos desensibilizantes y agente blanqueador asociado al uso de LED / láser (11), y Bonafe et al., el año 2014 usando la misma metodología en dientes con restauraciones anteriores, y concluyeron que el uso de estos agentes desensibilizantes antes del blanqueamiento reduce la intensidad de la sensibilidad (12).

También se ha estudiado la presencia de nitrato de potasio dentro de la composicion de los geles blanqueadores no reduce la sensibilidad dental. Maran et al., el año 2018 realizaron un ensayo clínico aleatorizado para evaluar el riesgo de sensibilidad dental y cambio de color después del blanqueamiento con un gel de peroxido de carbamida al $10 \%$ que contiene desensibilizante (nitrato de potasio al 3\% y fluoruro de sodio al $0.2 \%$ ) (13); cada participante del estudio uso el gel durante 3 horas durante 21 días, encontraron que la incorporación de nitrato de potasio y fluoru- ro de sodio en el gel blanqueador para el tratamiento ambulatorio no reduce la sensibilidad dental. Gallo et al., el año 2009 evaluaron el peróxido de carbamida al $30 \%$ con nitrato de potasio comparado con un control placebo (14). El tiempo de tratamiento para este estudio fue una hora por 10 días, para determinar si la mayor concentración del gel de blanqueamiento puede afectar la sensibilidad; concluyeron que el peróxido de carbamida al $30 \%$ con nitrato de potasio es eficaz para aclarar y tiene poco efecto en reducir la sensibilidad en comparación con el control, demostrando que es mejor el uso separado de este agente desensibilizante.

Por otro lado, Browning et al., el año 2008 realizaron un estudio en 22 pacientes comparando las diferentes concentraciones de nitrato de potasio: $3 \%, 0,5 \%$ agregados al gel de blanqueamiento de peróxido de carbamida al $10 \%$; y encontraron diferencia significativa de disminución de la sensibilidad en los grupos de menor concentración del nitrato de potasio $(0,5 \%)$ (9).

El nitrato de potasio también se puede encontrar en los dentífricos mostrando efectividad contra la sensibilidad en su uso. Thiesen et al., el año 2013 evaluaron si el uso de dentífricos desensibilizantes usados 15 días antes, durante y después del blanqueamiento de consultorio con peróxido de hidrógeno al 35\% podría eliminar o reducir la sensibilidad (15), fueron evaluados dentífricos con diferentes agentes activos: Colgate Total (flúor), Colgate Sensitive Pro-Alivio (arginina, carbonato de cálcico y flúor), Sensodyne ProNamel (nitrato de potasio al 5\% y flúor) y un placebo; los resultados mostraron una reducción de la sensibilidad significativa con el uso de Sensodyne ProNamel durante la primera semana del blanqueamiento, demostrando así la efectividad del nitrato de potasio dentro de los dentífricos.

En el metanalisis de Wang et al., del año 2015 se evaluó la eficacia del nitrato de potasio y floruro de sodio como agentes desensibilizantes durante el tratamiento de blanqueamiento, y concluyeron que ambos reducen la sensibilidad durante el tratamiento (16).

Parreiras et al., en el 2018, en un ensayo clínico aleatorizado, de boca dividida y triple ciego, evaluaron la eficacia de un gel desensibilizante que contenía nitrato de potasio $5 \%$ y glutaraldehído $5 \%$ antes del blanqueamiento en el consultorio con $35 \%$ de peróxi- 
do de hidrógeno(17), el tratamiento con desensibilizante o control con placebo se asignó en un diseño de boca dividida. Los geles desensibilizantes se aplicaron y mantuvieron en contacto con el esmalte dental durante 10 minutos, seguidos de 2 sesiones de blanqueamiento separadas por 1 semana. Concluyeron en que la aplicación de gel desensibilizante que contiene nitrato de potasio y glutaraldehído antes del blanqueamiento redujo el riesgo y la gravedad de la sensibilidad dental, sin alterar la efectividad del blanqueamiento (17).

En un estudio reciente, Martini et al., en un ensayo clínico aleatorizado, triple ciego, con diseño de boca dividida, evaluaron la aplicación de gel desensibilizante (nitrato de potasio al 2\%) antes y después del blanqueamiento en el consultorio sobre la sensibilidad dental; en un grupo, el gel desensibilizante se aplicó durante 10 minutos antes del peróxido de hidrógeno al 35\%, y luego la aplicación de gel placebo después (18); en el otro grupo, el gel desensibilizante se aplicó antes y después del procedimiento de blanqueamiento durante 10 minutos, y concluyeron que la aplicación del agente desensibilizante no influyó en la efectividad del blanqueamiento, sin embargo, no fue eficaz para reducir la sensibilidad, cuando se aplicó antes del procedimiento, o antes y después (18).

\section{b. Flúor}

Es un agente cuyo mecanismo de acción es la oclusión de los túbulos dentinarios con cristales (floruro cálcico), lo que reduce el diámetro del túbulo y la transmisión de estímulos. En relación a la presentación de este producto en odontología, estas van desde el contendido de $1400 \mathrm{ppm}$ hasta $12300 \mathrm{ppm}$. En la mayoría de reportes e investigaciones se encontró que el flúor gel a distintas concentraciones se encuentran asociados a diferentes agentes desensibilizantes ya mencionadas en las clasificaciones anteriores o evaluados solos como agente desensibilizante como el estudio realizado por Browing et al., el año 2010 donde se comparó la ocurrencia subjetiva de la sensibilidad dental asociado con blanqueamiento dental en pacientes que se cepillaban con una pasta dental con nitrato de potasio (Sensodyne Fresh Mint de GlaxoSmithKline) versus una pasta dental convencional con fluoruro (Crest Cavity Protection de Procter \& Gamble) antes y durante el blanqueamiento dental casero (19). Para el presente se realizó un estudio clínico aleatorizado, controlado, multicéntrico, de dos grupos paralelos, donde se comparó la aparición de la sensibilidad dental (mediante una escala analógica visual VAS y un cuestionario PSS) y el cambio de tono de color (blanqueamiento) con una guía clásica VITA (Vident ${ }^{\mathrm{TM}}$ ). Para el blanqueamiento se utilizó peróxido de hidrogeno al 14\% (Crest Whitestrips Supreme de Procter \& Gamble) durante 30 minutos dos veces al día con por lo menos un intervalo de 3 horas entre aplicaciones por 14 días y el cepillado de $1 \mathrm{~min}$ dos veces al día: mañana y tarde con cada pasta dental. La pasta que contenía solo flúor no tuvo reducción significativa de la sensibilidad. Por el contrario, el uso de una pasta de dientes con nitrato de potasio antes y durante el blanqueamiento dental tiene el potencial de reducir la sensibilidad.

\section{c. Gluma}

La presentación de este producto es en gel, presenta en su composición: hidroxietil metacrilato, gluteraldehido y agua purificada y pertenece al grupo de adhesivos dentales de tercera generación donde el glutaraldehido forma polímeros en forma de barrera uniéndose al colágeno de la dentina y formando un precipitado que impide el paso de estímulos a las fibras nerviosas previniendo el movimiento y los cambios osmóticos en los túbulos dentinarios y va penetrando hasta $200 \mu \mathrm{m}$ formando múltiples capas. Con respecto a su modo de empleo este elemento se coloca sobre las superficies dentales frotando durante 30 segundos y posteriormente se seca ligeramente. Dentro de las ventajas más resaltantes tenemos que es de fácil manejo, no necesita mezclar y reducción del dolor inmediato (20).

Ibraim y Banna el año 2001, compararon en un estudio in vivo el efecto de tres diferentes agentes desensibilizantes: Gluma Desensitizeen, Seal \& Protect y Barniz Fluorado en el manejo de la hipersensibilidad dental después de la aplicación de blanqueamiento dental con peróxido de hidrógeno al 38\%, el número de participantes fueron 27 individuos divididos en 3 grupos según el agente desensibilizante aplicado antes y después del blanqueamiento, la sensibilidad se evaluó mediante una escala de puntuación verbal (21); los tres agentes desensibilizantes fueron igualmente efectivos pero los desensibilizantes a base de resina (Gluma Desensitizeen, Seal \& Protect) duraron más tiempo en cuanto a su mecanismo de acción debido a que se forma un precipitado entre el grupo HEMA del agente resinoso y el colágeno del diente a diferencia 
de los agentes a base de fluoruro (Barniz Fluorado) donde su acción está relacionada directamente con la concentración y tipo de agente blanqueador.

Al evaluar la sensibilidad dental antes, durante y después del blanqueamiento en consultorio Deepak Mehta et al., el año 2013 evaluaron la aplicación tópica de GLUMA (glutaraldehído + HEMA fabricado por Heraeus Kulzer) en pacientes sometidos a blanqueamiento dental con peróxido de hidrógeno al 40 $\%$, para este estudio se seleccionaron 46 participantes (16 hombres y 30 mujeres, con edades comprendidas entre los 18-40 años de edad (22); se evaluó la intensidad del dolor a través de la escala visual-analógica (VAS) antes del blanqueamiento, durante y a los 5, 10, y 15 min y después a 1, 24, 48 h, y 1 semana. El presente ensayo clínico mostró que la utilización de un desensibilizante a base de Gluma antes durante y después del blanqueamiento mostró una significativa reducción de la sensibilidad comparado con un placebo mediante los mecanismos antes mencionados.

En otro estudio, Diniz et al., el año 2018, evaluaron el riesgo de sensibilidad después del blanqueamiento en el consultorio luego de la aplicación tópica de glutaraldehído a base de resina; treinta y tres pacientes fueron asignados aleatoriamente a un grupo experimental (Gluma) y placebo (23); el blanqueamiento se realizó con gel de peróxido de hidrógeno al 35\% (tres aplicaciones $\times 15$ minutos cada una) en dos sesiones, el Gluma y el placebo fueron aplicados durante un minuto antes de la aplicación de un gel blanqueador en el consultorio, y concluyeron que el Gluma no pudo reducir el riesgo de sensibilidad y el blanqueamiento no fue afectado por la aplicación del desensibilizante.

\section{d. Fosfato de Cálcio Amorfo (ACP)}

El fosfato de calcio amorfo se presenta en forma de cristales que se precipitan sobre la superficie y parte interna de los túbulos dentinarios reduciendo la sensibilidad al obliterarlos de manera rápida e inmediata. Éste desensibilizante se encuentra en el mercado dentro de los mismos productos blanqueadores, como en los geles a base de peróxido de carbamida.

Es un material inorgánico, amorfo, de fosfosilicato de calcio y sodio, diseñado a partir de una clase de materiales conocidos como vidrios bioactivos.

Para evaluar el efecto desensibilizante de un gel blanqueador a base de peróxido de carbamida (PC) al $16 \%$ con fosfato de calcio amorfo, en un estudio
Giniger et al., el año 2005 evaluaron a 50 participantes que fueron distribuidos en dos grupos para utilizar en forma aleatorizada el gel que contiene ACP o el gel control PC 16\% sin desensibilizante (24). Ambos grupos utilizaron sus respectivos productos durante tres horas al día por 14 días y realizaron mediciones clínicas doble ciego en los días tres, siete, catorce y el quinto día post-tratamiento. El grupo asignado con PC $16 \%+$ ACP demostró significativamente menor sensibilidad térmica tanto durante como después del tratamiento en comparación al grupo control y ambos grupos demostraron similar efecto blanqueador.

Por otro lado encontramos el fosfato de calcio unido a iones de silicato y sodio que presentan el mismo mecanismo del fosfato de calcio amorfo presentando el mismo mecanismo de acción se presenta en dentífricos los cuales también son efectivos para disminuir la sensibilidad, Kakodkar et al., el año 2013 realizaron un estudio in vitro en 30 premolares para evaluar la oclusión del túbulo dentinal bajo la acción de la pasta dental desensibilizante NovaMin combinado con 15\% de peróxido de carbamida (propoción1:1) (25); las piezas fueron seccionadas para ser observadas a través de un microscopio de barrido y se contabilizó el número de túbulos que fueron obliterados por completo o parcialmente así como los túbulos abiertos de cada espécimen, se encontró que la combinación utilizada ocluye los túbulos dentinarios sin afectar el resultado del blanqueamiento.

Para evaluar otros tipos de compuestos formulados base de los cristales de calcio Kossatz et al., evaluaron la sensibilidad dental al utilizar peróxido de hidrógeno al $35 \%$ con gluconato de calcio al $2 \%, 40$ participantes divididos en 2 grupos: el primer grupo recibió peróxido de hidrógeno al $35 \%$ sin calcio y el segundo grupo peróxido de hidrógeno al 35\% con calcio durante dos semanas, para la evaluación de la sensibilidad se utilizó una escala del 0 al 4, demostraron que el grupo de peróxido de hidrógeno al $35 \%$ con gluconato de calcio al $2 \%$ presentó menor sensibilidad dental durante el blanqueamiento dental en el consultorio (26). Por otro lado Loguercio et al., mediante un ensayo clínico encontraron que la aplicación de una pasta con nanopartículas de fosfato de calcio durante el blanqueamiento dental no reduce la sensibilidad causada por el blanqueamiento dental (27).

Alexandrino et al., en un estudio clínico aleatorizado, controlado y doble ciego evaluaron el efecto del 
fosfosilicato de sodio y calcio (NovaMin) y el fosfato de calcio amorfo fosfopeptídico de caseína con fluoruro (CPP-ACPF), en la prevención de la sensibilidad postoperatoria y los efectos del tratamiento de blanqueamiento clínico. Los participantes fueron tratados con peróxido de hidrógeno al 35\%, un grupo fue tratado con NovaMin y el otro con CPP-ACPF. Ambos agentes bioactivos fueron aplicados durante cinco minutos. Concluyeron que la combinación de CPP-ACPF con peróxido de hidrógeno al 35\% redujo significativamente la sensibilidad postoperatoria en la primera sesión, en comparación con los otros tratamientos evaluados, la asociación de CPP-ACPF y NovaMin no afectó el cambio de color causado por el blanqueamiento dental (28).

El fosfato de calcio de amorfo puede ser añadido a los geles blanqueadores sin que éste afecte su efectividad disminuyendo la sensibilidad dentinaria.

\section{e. Hidroxiapatita}

Las nano-partículas de hidroxiapatita reponen mineral perdido durante el blanqueamiento, se adhieren y ayudan a eliminar la placa bacteriana, reparan las fisuras microscópicas de la superficie, remineralizan zonas desmineralizadas y ocluyen los túbulos dentinarios expuestos, este agente se puede encontrar en pastas dentales y pastas de pulido.

Se ha demostrado que este producto puede reducir la sensibilidad durante y después del blanqueamiento como en el estudio de Browning et al., quienes evaluaron el uso de una pasta de nano-hidroxiapatita Renamel After Bleach ${ }^{\circledR}$ (Sangi Co., Ltd., Tokio, Japón) en la reducción de la sensibilidad y si afecta su eficacia durante blanqueamiento dental, a un primer grupo se le aplicó esta pasta y al segundo grupo un placebo durante y después del blanqueamiento con peróxido de hidrógeno al $7 \%$, la sensibilidad se evaluó mediante una escala visual análoga, la utilización de esta pasta se asoció con una reducción en el número de días de sensibilidad dental experimentada durante el blanqueamiento (29).

El uso de esta pasta disminuyo la intensidad se recomienda su aplicación durante y después del blanqueamiento dental.

\section{f. Antiinflamatorios}

Se ha reportado el uso de antiinflamatorios no esteroideos (AINES) para reducir la sensibilidad por blanqueamiento dental, como el Ibuprofeno que inhibe la síntesis y liberación de prostaglandinas en los tejidos corporales por inhibición de la ciclooxigenasa 1 y 2 (COX1 y COX2) se pueden encontrar en tabletas de 200,400, 600 y $800 \mathrm{mg}$.

Se ha demostrado que el Ibuprofeno solo tiene acción limitada en la sensibilidad hasta 1 hora después de realizado el blanqueamiento dental como lo observaron Charakorn et al., evaluaron el efecto de este fármaco en la sensibilidad dental asociada al blanqueamiento con peróxido de hidrógeno al 38\% (Opalescence Xtra Boost, Ultradent Products, Inc., South Jordan, UT, EE.UU.) en 31 pacientes, el primer grupo utilizó un placebo y el segundo ibuprofeno de 600 mg (Advil Liquid Gel, Wyeth, Madison, NJ, EE.UU.) tomaron las pastillas 30 minutos antes del tratamiento blanqueador, para la evaluación del dolor se utilizó una escala visual análoga y se encontró que el ibuprofeno ayudó a reducir la intensidad sensibilidad dental hasta una hora después del blanqueamiento en consultorio (30).

Del mismo modo, De Paula et al., evaluaron el efecto de la administración preoperatoria de ibuprofeno de $400 \mathrm{mg}$ en la sensibilidad dental causada por el blanqueamiento con peróxido de hidrógeno al 35\% (Whiteness HP Maxx FGM, Joinville, SC, Brazil), en 30 adultos que recibieron un placebo o ibuprofeno 1 hora antes del blanqueamiento y otras dosis cada ocho horas durante 2 días (31); la sensibilidad se registró 48 horas después del tratamiento a través de dos escalas: visual análoga y calificación verbal, se observó menor sensibilidad dental en el grupo experimental sólo hasta una hora post-blanqueamiento, el resto de tiempo se observó similar sensibilidad dental.

Estos mismos autores evaluaron también el etoricoxib como agente antiinflamatorio antes del blanqueamiento(31). El etoricoxib es un inhibidor selectivo de la ciclooxigenasa 2 (COX-2), que se cree que está implicado en la respuesta inflamatoria y ha sido considerada responsable de la producción de prostaglandinas, se encuentra en presentaciones de 60, 90, $120 \mathrm{mg}$. Los autores evaluaron el efecto del etoricoxib de $60 \mathrm{mg}$ en la sensibilidad dental causada por el blanqueamiento con peróxido de hidrogeno al 35\% (Whiteness HP Maxx FGM, Joinville, SC, Brazil), y no encontraron diferencias significativas en la prevalencia y la intensidad de la sensibilidad dental con el uso de este fármaco, el uso de etoricoxib no reduce la 
experiencia ni la intensidad de la sensibilidad dental (32).

Vaez et al., determinaron la efectividad de la administración preventiva de etodolac sobre el riesgo y la intensidad de la sensibilidad dental, seleccionaron cincuenta pacientes para este ensayo clínico triple ciego, aleatorizado, cruzado y controlado con placebo. Etodolac $(400 \mathrm{mg})$ o placebo se administró en una dosis única 1 hora antes del procedimiento de blanqueamiento; la sensibilidad dental se evaluó antes, durante y 24 horas después del procedimiento. La administración preventiva de una dosis única de $400 \mathrm{mg}$ de etodolac no afectó el riesgo de sensibilidad dental ni el nivel de sensibilidad informado por los pacientes, durante o después del procedimiento de blanqueamiento dental en el consultorio (33).

Con la presente revisión concluimos que no se recomienda el uso de antiinflamatorios debido a su limitada acción después del blanqueamiento.

\section{g. Antioxidantes}

En la literatura se encontraron estudios que evalúan el uso de antioxidantes para eliminar la sensibilidad por blanqueamiento como el De Paula et al., quienes evaluaron el efecto del ácido ascórbico de $500 \mathrm{mg}$. (píldora de vitamina C, Citroplex, Laboratorio Neo Química, Anápolis, GO, Brasil) en la sensibilidad inducida por blanqueamiento dental, en este estudio se evaluaron a 39 participantes los cuales fueron divididos aleatoriamente en 2 grupos: un grupo recibió un comprimido de ácido ascórbico y el otro un placebo. Las dosis se administraron tres veces al día durante 48 horas, la primera dosis se administró una hora antes de cada sesión de blanqueamiento; se realizaron dos sesiones de blanqueamiento con peróxido de hidrógeno al 35\% con una semana intervalo (34); la sensibilidad dental se registró hasta las 48 horas después del blanqueamiento. Ambos grupos mostraron similar sensibilidad dental. Concluyendo que el uso de un antioxidante como el ácido ascórbico $(500 \mathrm{mg}$, tres veces al día) no es capaz de prevenir la sensibilidad dental o reducir su intensidad.

\section{h. Láser LED}

El equipo hibrido con LED y láser de baja intensidad se ha convertido en un método sugerido para mejorar la eficacia del blanqueamiento dental y controlar la sensibilidad, como una ayuda auxiliar analgésica inmediata para la repolarización de la membrana nerviosa alterada. Sin embargo, existen pocos estudios clínicos que evalúen la eficacia del láser LED, como el De Almeida et al., quienes evaluaron la eficacia del blanqueamiento en consultorio y el control de la sensibilidad con tratamiento láser LED, dispositivo de origen híbrido (Whitening Laser II, DMC Equipamientos LTDA, Sao Carlos, SP, Brasil) (35), en el estudio se utilizó seis LED azules $(425-480 \mathrm{~nm})$ con salidas de potencia $300 \mathrm{~mW}$ cada uno, y tres lásers infrarrojos $(810 \mathrm{~nm})$ con poder $200 \mathrm{~mW}$. El sistema de irradiación láser LED fue de $300 \mathrm{~mW} / \mathrm{cm}^{2}$ por diente. A ambos se les realizó los blanqueamientos con peróxido de hidrógeno al $35 \%$, al primer grupo sólo se le activo con luz LED y el segundo fue activado por láser LED. En ambos grupos la luz se activó tres veces: 1 minuto de activación, intercalados de dos minutos con el gel en reposo, después de la tercera aplicación del gel permaneció por tres minutos y fue eliminado. Le gel fue reemplazado, y el protocolo se repitió dos veces más con un total de 30 minutos, los tratamientos fueron realizados durante dos sesiones con intervalos de 1 semana entre sesiones y 2 semanas entre tratamientos. La sensibilidad dental fue similar para ambos grupos en diferentes periodos. Concluyendo que el uso de láser LED no es capaz de prevenir o reducir la sensibilidad dental.

\section{CONCLUSIONES}

Las terapias desensibilizantes revisadas han demostrado disminución en la duración e intensidad de la sensibilidad causada por blanqueamiento dental y limitan los cambios en la morfología del esmalte y la dureza de la superficie dental causada por el tratamiento.

La inclusión de diferentes agentes desensibilizantes en los geles de blanqueamiento dental no alteran su efectividad y permitirían que el paciente continúe y complete el tratamiento.

De las diferentes terapias desensibilizadoras el nitrato de potasio en gel ha ofrecido mejores resultados al aplicarlo antes del blanqueamiento dental.

\section{Correspondencia:}

Lidia Yileng Tay Chu Jon

Correo electrónico: lidia.tay.c@upch.pe 


\section{REFERENCIAS BIBLIOGRÁFICAS}

1. Mondelli RFL. Clareamento de dentes polpados: Técnicas e equipamentos. Rev Odont. Biodonto.2003; 1(1):10-71.

2. Araujo FO, Baratieri LN, Araújo E. In situ study of in-office bleaching procedures using light sources on human enamel microhardness. Oper Dent. 2010; 35(2):139-46.

3. Joiner A. The bleaching of teeth: a review of the literature. J Dent. 2006; 34(7):412-9.

4. Leonard RH Jr, Haywood VB, Phillips C. Risk factors for developing tooth sensitivity and gingival irritation associated with night guard vital bleaching. Quintessence Int. 1997; 28(8):527-34.

5. Hoi L P, Wilson N. Effects of different desensitizing agents on bleaching treatments. Eur J of General Dentistry. 2014; 3 (2): 93- 9.

6. Jacobsen PL, Bruce G. Clinical dentin hypersensitivity: understanding the causes and prescribing a treatment. J Contemp Dent Pract. 2001; 2(1):1-12.

7. Li Y, Greenwall L. Safety issues of tooth whitening using peroxide-based materials. Br Dent J. 2013; 215(1):29-34.

8. Po LH, Wilson NW. Effects of different desensitizing agents on bleaching treatments. Eur J Gen Dent. 2014;3:93-9.

9. Browning WD, Chan DC, Myers ML, Brackett WW, Brackett MG, Pashle DH. Comparison of Traditional and Low Sensitivity Whiteners. Oper Dent. 2008; 33(4): 379-85.

10. Tay LY, Kose C, Loguercio AD, Reis A. Assessing the effect of a desensitizing agent used before in-office tooth bleaching. J Am. Dent Assoc. 2009;140(10):124551

11. Reis A, Dalanhol AP, Cunha TS, Kossatz S, Loguercio AD. Assessment of tooth sensitivity using a desensitizer before light-activated bleaching Oper Dent. 2011; 36(1): 12-7.

12. Bonafé E, Loguercio AD, Reis A, Kossatz S. Effectiveness of a desensitizing agent before in-office tooth bleaching in restored teeth. Clin Oral Investig. 2014; 18(3):839-45.

13. Maran BM, Vochikovski L, de Andrade Hortkoff DR, Stanislawczuk R, Loguercio AD, Reis A. Tooth sensitivity with a desensitizing-containing at-home bleaching gel-a randomized triple-blind clinical trial. J Dent. 2018; 72:64-70. doi: 10.1016/j. jdent.2018.03.006

14. Gallo J, Burgess J, Ripps H, Bell J, Mercante D, Davidson J. Evaluation of $30 \%$ carbamide peroxide at-home bleaching gels with and without potassium nitrate - A pilot study. Quintessence Publishing. 2009; 40(4): 1-6.

15. Thiesen $\mathrm{CH}$, Rodrigues R, Maykot LH, Sartori N.
The influence of desensitizing dentifrices on pain induced by in-office bleaching. Braz Oral Res. 2013; 27(6):517-23.

16. Wang Y, Gao J, Jiang T, Liang S, Zhou Y, Matis BA. Evaluation of the efficacy of potassium nitrate and sodium fluoride as desensitizing agents during toothbleaching treatment-A systematic review and meta-analysis. J Dent. 2015;43(8):913-23.

17. Parreiras SO, Szesz AL, Coppla FM, Martini EC, Farago $\mathrm{PV}$, Loguercio $\mathrm{AD}$, Reis A. Effect of an experimental desensitizing agent on reduction of bleaching-induced tooth sensitivity: A triple-blind randomized clinical trial. J Am Dent Assoc. 2018;149(4):281-290. doi: 10.1016/j.adaj.2017.10.025

18. Martini EC, Parreiras SO, Szesz AL, Coppla FM, Loguercio $\mathrm{AD}$, Reis $\mathrm{A}$. Bleaching-induced tooth sensitivity with application of a desensitizing gel before and after in-office bleaching: a triple-blind randomized clinical trial. Clin Oral Investig. 2019.1:1-10. doi: 10.1007/s00784-019-02942-9

19. Browning W, Haywood V, Hughes N, Cordero R. Prebrushing with a Potassium nitrate dentifrice to reduce tooth sensitivity during bleaching evaluated in a practice-based setting. Compendium. 2010; 31(3): 220- 5.

20. Schüpbach P, Lutz F, Finger W. Closing of dentinal tubules by Gluma desensitizer. Eur J Oral Sci. 1997; 195: 414-21.

21. Mohamed A, Ibrahim, Mai E. Evaluation of the sustainability of Different Desensitizing Agents after in-Office Bleaching. Life Science Journal. 2011; 8(1):164-8.

22. Deepak M, Suresh V, Meena N, Linga R, Usha. Clinical trial of tooth desensitization prior to in office bleaching. European Journal of Oral Sciences. 2013; 121(5): 477-81.

23. Diniz A, Lima S, Tavarez R, Borges AH, Pinto S, Tonetto MR, Loguercio AD, Bandéca MC. Preventive use of a resin-based desensitizer containing glutaraldehyde on tooth sensitivity caused by in-office bleaching: A randomized, single-blind clinical trial. Oper Dent. 2018; 43(5):472-481. doi: 10.2341/17-020-C

24. Giniger M, Macdonald J, Ziemba S, Felix H. The clinical performance of professionally dispensed bleaching gel with added amorphus calcium phosphate. J Am Dent Assoc. 2005, 136(3):383-92.

25. Kakodkar G, Lavania A, Ataide Ide N. An in vitro SEM study on the effect of bleaching gel enriched with Nova Min on whitening of teeth and dentinal tubule occlusion. J Clin Diagn Res. 2013; 7(12):3032-5.

26. Kossatz S, Martins G, Loguercio AD, Reis A. Tooth sensitivity and bleaching effectiveness associated with use of a calcium-containing in-office bleaching gel. J Am Dent Assoc. 2012; 143(12):81-7.

27. Loguercio AD, Tay LY, Herrera DR, Bauer J, Reis A. Effectiveness of nano-calcium phosphate paste on 
sensitivity during and after bleaching: a randomized clinical trial. Braz Oral Res. 2015;29:1-7.

28. Alexandrino LD, Alencar CM, Silveira ADSD, Alves $\mathrm{EB}$, Silva CM. Randomized clinical trial of the effect of NovaMin and CPP-ACPF in combination with dental bleaching. J Appl Oral Sci. 2017;25(3):335-340. doi: 10.1590/1678-7757-2016-0408

29. Browning WD, Cho SD, Deschepper EJ. Effect of a nano-hydroxyapatite paste on bleaching-related tooth sensitivity. J Esthet Restor Dent. 2012; 24(4):268-76.

30. Charakorn P, Cabanilla LL, Wagner WC, Foong WC, Shaheen J, Pregitzer R, et al. The effect of preoperative ibuprofen on tooth sensitivity caused by in-office bleaching. Oper Dent. 2009; 34(2):131-5.

31. De Paula EA, Loguercio AD, Fernandes D, Kossatz S, Reis A. Perioperative use of an anti-inflammatory drug on tooth sensitivity caused by in-office bleaching: a randomized, triple-blind clinical trial. Clin Oral Investig. 2013; 17(9):2091-7.

32. De Paula EA, Kossatz S, Fernandes D, Loguercio A, Reis A. The effect of perioperative Ibuprofen use on tooth sensitivity caused by in-office bleaching. Oper Dent. 2013; 38(6):601-8.

33. Vaez SC, Faria-E-Silva AL, Loguércio AD, Fernandes MTG, Nahsan FPS. Preemptive use of etodolac on tooth sensitivity after in-office bleaching: a randomized clinical trial. J Appl Oral Sci. 2018;26:e20160473.

34. De Paula EA, Kossatz S, Fernandes D, Loguercio AD, Reis A. Administration of ascorbic acid to prevent bleaching-induced tooth sensitivity: a randomized triple-blind clinical trial. Oper Dent. 2014; 39(2):12835.

35. De Almeida Farhat PB, Santos FA, Gomes JC, Gomes OM. Evaluation of the efficacy of LED-laser treatment and control of tooth sensitivity during in-office bleaching procedures. Photomed Laser Surg. 2014; 32(7):422-6.

Recibido: 14-06-2018

Aceptado: 05-10-2019 